\title{
Panel Report on
}

\section{THEORY OF SURFACES}

\section{MASTER}

Published Under the Auspices of the

COUNCIL ON MATERIALS SCIENCE

for the

DIVISION OF MATERIALS SCIENCES

U. S. DEPARTMENT OF ENERGY

JANUARY, 1979 


\section{DISCLAIMER}

This report was prepared as an account of work sponsored by an agency of the United States Government. Neither the United States Government nor any agency Thereof, nor any of their employees, makes any warranty, express or implied, or assumes any legal liability or responsibility for the accuracy, completeness, or usefulness of any information, apparatus, product, or process disclosed, or represents that its use would not infringe privately owned rights. Reference herein to any specific commercial product, process, or service by trade name, trademark, manufacturer, or otherwise does not necessarily constitute or imply its endorsement, recommendation, or favoring by the United States Government or any agency thereof. The views and opinions of authors expressed herein do not necessarily state or reflect those of the United States Government or any agency thereof. 


\section{DISCLAIMER}

Portions of this document may be illegible in electronic image products. Images are produced from the best available original document. 
Panel Report on

THEORY OF SURFACES

Published Under the Auspices of the

COUNCIL ON MATERIALS SCIENCE

for the

DIVISION OF MATERIALS SCIENCES

U. S. DEPARTMENT OF ENERGY

JANUARY, 1979

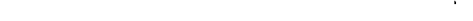

Published by University of IIlinots Under Contract DE-AS02-76ER01798. 


\section{PREFACE}

This report is one of a series resulting from studies conducted under the auspices of the Department of Energy (DOE) Council on Materials Science. The Council was established to provide the DOE Division of Materials Sciences with in-depth studies of selected important areas of materials research and was an outgrowth of a series of overview workshops held in the Spring of 1977. The workshops were held to lay the foundation for basic materials research needs and opportunities for the then impending new energy departinent. Reports of these workshops were subsequently published. Professor Maurer of the University of 111 inois is the current Council Director.

During 1.978, the Council on Materials Sctence sponsored two studies: I. High Temperature Ceramics, Chaired by Professor Kent Bowen of MIT and II. Theory of Surfaces, Chaired by Dr. Don Hamann of Bell Laboratories. The Pane1. Chairmen selected the Panel members who met during the summer of 1978 for about a week each. Preliminary results were presented to the Council and the Materials Sciences Division staff in September, 1978.

This report and others in the series will serve both the DOE. Division of Materials Sciences and the scientific community in providing guidance for research directions. It is expected that the Council will sponsor about two studies each year and in addition, the Council will meet for a week-long session each sunmer to discuss the year's activities and explore new concepts. 
Panel on Theory of Surfaces

D. R. Hamann, Be 11 Laboratories, Chairman

W. L. Clinton, Georgetown University \& Division of Materials Sciences

J. D. Doll, State University of New York

L. M. Falicov, University of California

P. J. Feibelman, Sandia Laboratories

.T. E. Fischer, Exxon Corporate Research Lab

J. D. Joannopoulos, Massachusetts Institute of Technology

F. Jona, State University of New York

L. Kleinman, University of Texas

R. P. Messmer, General Electric Company

D. L. Mills, University of California

E. W. Plummer, University of Pennsylvania

M. A. Schluter, Bell Laboratories

P. P. Schmidt, Qakland University

W. E. Spicer, Stanford University

H. Suhl, University of California

S. Y. Tong, University of Wisconsin

Council on Materials Science:

Robert J. Maurer, University of Illinois, Director, CMS

Philip W. Anderson, Bell Telephone Laboratories

Robert W. Balluffi, M.I.T.

W. Dale Compton, Ford Motor Company

Brian R. I. Frost, Argonne National Laboratory

W. David Kingery, M.I.T.

David A. Shirley, University of California-Berkeley

John C. Wheatley, University of California-San Diego

DOE Representatives:

William L. Ciinton, UMS \& Georgetown Untversity

Louis C. Ianniello, Chief, Metallurgy \& Ceramics Branch, DMS

Donald K. Stevens, Director, DMS 
Council on Materials Sciences Panel on

SURFACE THEORY

14-18 August 1978

D. R. Hamann, Chairman

Bell Laboratories

Murray Hill, New Jersey 07974

W. L. Clinton

Division of Materials Science

Department of Energy

Washington, DC 20545

J. D. Doll

Department of Chemistry

State University of New York

Stony Brook, New York 11974

L. M. Falicov

Department of Physics

University of California

Berkeley, California 94720

P. J. Feibelman

Sandia Laboratories 5151

Albuquerque, New Mexico 87115

T. E. Fischer

Exxon Corporate Research Lab

P. O. Box 45

Linden, New Jersey 07036

J. D. Joannopoulos

Department of Physics

Mass. Inst. of Technology

Cambridge, Massachusetts 02139

F. Jona

Department of Materials Science

State University of New York

Stony Brook, New York 11794

L. Kleinman

Department of Physics

University of Texas

Austin, Texas 78712
R. P. Messmer

General Electric Company

Research \& Development Center

Schenectady, New York 12301

D. L. Mills

Department of Physics

University of California

Irvine, California 92717

E. W. Plummer

Department of Physics

University of Pennsyivania

Philadelphia, Pennsylvania

19174

M. A. Schluter

Bell Laboratories

Murray Hill, New Jersey 07974

P. P. Schmidt

Department of Chemistry

Oakland University

Rochester, Michigan 48063

W. E. Spicer

Stanford Electronics Lab

Stanford University

Stanford, California 94305

H. Suhl

Department of Physics

University of California

La Jolla, California 92093

S. Y. Tong

Department of Physics

University of Wisconsin

Milwaukee, Wisconsin 53201 
CONTENTS

I. Introduction

II. Purpose and Scope

III.: Role of Theory

A. General Role of Theory

B. Classification of Theory

C. The Science-Technology Interface

D. An Example

IV. Introduction to problem areas

V. Problem Areas Focusing on Atoms

A. LEED Structure Determinations

B. Atom Diffraction

C. Surface Vibrations: Intrinsic Properties and Experimental Probes

D. Interactions of Molecular Beams with Surfaces

E. Reaction Kinetics Over Surfaces

F. Interface Transport properties

G. Adsorbate-Adsorbate Interactions

H. Precursors

I. Reconstruction Mechanisms

VI. Problem Areas Focusing on Electrons

A. Semiconductor and Simple Metal Electronic structure -Pseudopotential Calculations

B. Semiconductor Surface Electronic Structure -- Empirical calculations

C. Transition Metal Surfaces -- First principles Calculations

D. Transition Metal Surfaces -- Empirical Calculations

E. Cluster Models for Electronic Structure

F. Electron-Surface Scattering

G. Many-Electron Effects

H. Photoemission Theory

I. Core Level excitations

J. Extended vs Localized Electron States

K. Positron-Surface Interactions

VII. Froblem Areas - Generäl

A. Solid-Electrolyte Interfaces

B. Alloy Surfaces

C. Electron-Stimulated Desorption

D. Empirical Modeling of Chemical Trends in Surface Phenomena

VIII. Innovation in surface science

IX. Institutional Issues

A. Basic vs Applied Research

B. Promotion of Innovation

C. Supply of Young Scientists

D. New Surface science centers

E. Critical Mass and communication

F. Surface Theory Outside the USA 
In March of 1978, a Resource Council for Materials Science was established to provide a forum for assessment and stimulation of research areas fertinent to the mission of the Materials Science program of the Basic Energy Science Division. us Department of Energy. The council, consisting at its initial meeting of a seven member steering committee, selected the Theory of surfaces as a study area to be reviewed by a panel of scientists during the summer of 1978. This is the report of the panel formed for that purpose.

The panel was organized by the chairman following guidelines suggested by the steering committee. It consisted of 17 scientists active in surface research, including 13 theorists and 4 experimentalists. The research interests of the panel members span a broad range of topics, with some degree of emphasis on topics that have developed rapidly in recent years.

The panel met for four and one-half days, 14-18 August 1978. in Hyannis. Massachusetts. Each panelist had been asked to prepare a talk reviewing recent progress and outstanding problems in his area of interest. These talks occupied the first two days of the meeting. with considerable time allotted for discussion between talks. On the third day. the group as a whole spent a full session prefaring a list of problem areas. and a partial session assigning groups of two to fuir panelists to prepare write-ups on each area. The panel spent the remainder of the third day and much of the fourth day in small groups discussing and preparing the write-ups. The firal session of the entire panel was held on the evening of the fourth day. and devoted to a discussion of innovation in surface theory and institutional issues. Problem area write-ups were complcted on the morning of the fifth day.

'We purpose aind scope of the panel are discussed in section II. A considerable amount of discussion during the first two days of meetings was devoted to examining the role played by theory in surface science and in the application of surface science to energy technology. A summary of the panel's conclusions is given in section III. The selection of 24 problem areas and the implications of the selection process is discussed in section IV. Sections V. VI and VII contain the detailed discussion of each problem area, very roughly grouped. These constitute the bulk of the specific technical recommendations of the report. Conclusions of the pane $1^{\circ} \mathrm{s}^{\mathrm{d}} \mathrm{s}$ scussions of innovation and of institutional issues are summarized in sections VIII and IX. 
II. PURPOSE AND SCCPE

In the spring of 1977, a series of nine workshops was organized to study the complete range of research activities supported under the Materials Sciences Program and the relation of these activities to the needs of energy technology. Surface science was the topic of one of the workshops. and the report of this workshop repeatedly stressed the need for theoretical contributions. Several other workshops also reported a need for experimental and theoretical surface research within the materials areas they studied.

The surface science workshof divided its studies into two major topics: the state of surface science as related to energy technologies and surface science directions and goals. This information is still current, and this panel has not attempted to duplicate the detailed relationships between surface science and energy technologies contained in the workshop report.

Since the scope of the surface science workshop was very broad, and only four of 50 participants were theorists, very little detail was given as to the nature, feasibility or relative importance of theoretical contributions. The overall purpose of the present surface Theory Panel is to provide depth and focus in this area. In particular, the panel sought answers to the following questions:

- What is the status of active areas of current theoretical research?

- What is important?

- What is likely to become important?

- What is the role of theory in surface science?

- What is the role of theory in interfacing surface science with technology?

The scope of the panel's considerations was as broad as possible within the limitations of a discussion group of workable size and duration. The following de facto limitations of scope were clear to the panelists, and should be borne in mind by the reader:

- This report is not a review article. The authors of somie subject area discussions chose to include a few strictly illustrative references to the literature, while others did not.

- Areas of surface science where there is little or no existing or anticipated theoretical work are not covered 


$$
-4-
$$

- Complex surface processes such as catalysis or corrosion are not treated as separate subjects. The manner in which theories of simpler phenomena bears on them is noted where possible. 
III. ROLE OF THECRY

\begin{abstract}
Surface science has been, by tradition, primarily an experimental field. It is an old field, and was interdisciplinary long before the term became fashionable. Early contributions by theorists were few in number and qualitative in nature, such as Bardeen's theory of work functions or Shockley"s of surface states. This is not to say that there was no theoretical understanding of surface phenomena. concepts from solid-state physics and from chemistry were adopted for qualitative and semiquantitative interpretation of surface experiments, but this was primarily done by the experimentalists themselves. starting around 1970. an increasing number of theorists began to enter the surface field from backgrounds in solid-state theory and quantum chemistry. This trend was spurred in part by the increasing availability of reproducible experimental data on simple well-characterized surface systems, and in part by the realization that surface theory had been left far behind during the rapid development of bulk theory in the $50^{\circ} \mathrm{s}$ and $60^{\circ} \mathrm{s}$. The rapidly developing body of theory of this recent period has involved a great deal of interaction and cross-stimulation between experimental and theoretical research, and it is theoretical research of this nature which frimarily occupied the panel.
\end{abstract}

A. General Role of Theory

The first step in approaching a problem in surface science is to abstract a simple system from the "real" problem of ultimate interest. For example, to begin to study a certain class of catalytic reactions, one might start with an ordered layer of one reactant on a single crystal surface. The selection of systems for experimental study is primarily the task of the experimentalist, but it is often strongly influenced. by theorists in a feedback loop.

The basic task of theory in surface science (or any other science for that matter) is:

- To unify and relate results of many experiments

These may be:

- Different measurements on one system

- one measurement on a family of systems

The above broad goals are the underlying and generally unstated goals of all theoretical research. The distinction between interpretive and predictive theory is not a real one. When stimulated by experimental data, theory is, if successful, interpretive. When the same theory succeeds for a number of systems and gains credibility. it becomes predictive. The 
process of experiment stimulating theory stimulating new experimental tests stimulating refinement of the theory, etc.. is the essential "feedback loop" mechanism which is promoting rapid progress in a number of areas of surface science at this time. While this process exists in any area of science. it was a strongly held conviction of the panel that:

- close coupling of theory and experiment is extremeiy important in surface science.

B. Classifications of Theory

There are many ways in which theoretical research can be classified. Two proved particularly useful to the panel. The first tyce of distinction separates:

- Theory describing the intrinsic behavior of a system

- Theory treating the interaction of an experimental probe with systems.

While this distinction cannot always be cleanly made, it is an especially pertinent one in surface science. The probes which are most surface sensitive (e.g.. low-energy electron beams and atom beams): are surface sensitive precisely because they interact strongly with the surface and near-surface region of the system under study. The strong interaction renders the task of extracting buried information about the system itself (e.g.. atomic geometry and elementary excitations) highly nontrivial. While in principle the problem of each type of experimental probe interacting with each system of interest could be treated separately, it is of great practical advantage to have one set of theoretical tools tied to particular experimental probes, and another set to the intrinsic properties of the systems themselves. Most of the problem areas discussed fall clearly in one of these categories or another.

Another useful class of distinctions separates different "levels" of theory: possible)

- Firbt-principles theories (keep as mush detail. as

- Model theories (discard as much detail as possible)

- Empirical theories (aiscard the model).

While there is a clear logical hierarchy in this classification, an attendent value judgment would be badly misplaced.

Ideally. the development $c f$ a new area in theoretical surface science would proceed as follows: First-principles calculations retaining as complete a description of the system as 
possible would be carried out for a few carefully selected systems. Aspects of the results could be tested directly against experiment, and the calculations could then serve to develop intuition about the class of problems under study, and as benchmarks to test model calculations. Model theories. developed using the results of the first-principles calculations for guidance. could then simplify the computations sufficiently that a much larger group of more complex situations could be studied. Finally, regularities discovered in the course of studying a large number of systems could be systematized into a set of formulas or rules capable of predicting significant quantitative asfects of the phenomena of interest directly from other physical or chemical properties.

In practice, the development of a new area seldom proceeds as outlined above. It may be entered at any of the three levels, and information and motivation may flow in either direction. A good empirical formula, for example, coula certainly stimulate attempts to model the underlying processes. A hallmark of the recent era of development in surface theory has been the interaction between first-principles and model theories. Much work prior to this suffered from oversimplification of models to the extent that meaningful interaction with experimental research was not cossible.

A type of distinction which has not proved useful in the panel's discussions is that of "conceptual" versus "computational" advances. The identification of mechanisms in various surface phenomena goes hand in hand with the development of models and computational verification by comparison with experiment. The kind of advances achieved in some areas of solidstate physics by applying new mathematical sophistication to long-existing. simple models has not had any parallel in surface science.

\section{The science-Technology Interface}

There is an extremely simple and direct argument which indicates the important role theory should play in the interface of surface science and technology: mechanisms and models can be utilized at one atmosphere of pressure, while most surface science experimental techniques cannot. It is not to be expected. of course. that a calculation incorporating a sufficient number of details to completely characterize a practical technical process could be carried through. However, a complete treatment is rot necessary for theoretical results to be of value. Most development of new materials and surface treatmerts for technological application will continue to be done by intuition-guided cut-and-try techniques. Theoretical contribu= tions can:

- Focus a cut-and-try search in the most profitable direc- 
- Guide intuition development by providing models and mechanisms.

Experimental surface science methods are, in fact, widely used as diagnostic tools in applied research. Since a significant fraction of theoretical effort in surface science concerns interpretation of such data, a third effect on technological development is

- Improved data interpretation.

The question of which theoretical problem areas in surface science can contribute most directly to which areas of enerqy technology is, in most regards, not a valid one. In most areas where surtace scituce may bc experter to have an impact, areas as disfarate as corrosion and catalysis, the same elementary surface crocesses are involved.

D. An Example

A case history of development and application by the Surface Physics Department of the Exxon Corporate Research Laboratory was reported to the panel by $T$. Fischer. Since it bears heavily on the role of theory in particular and surface science in general in energy technology, it is summarized here.

Awareness of the importance of alloy catalysts in the petroleum industry, and the importance of surface segregation on the behavior of alloy catalysts led this grouf to undertake a systematic experimental study of the poorly understood segregation problem. After sufficient data were gathered, an empirical theory relating the surface concentration and its temperature dependence to particular features of the phase diagram of bulk binary alloys was devised. Shortly thereafter, the group became aware of a pressing metallurgical problem in their industry: hydrogen-sulphide embrittlement of steel in deep well arilling. There is a known analogy between grain boundaries and surfaces. and a known influence of grain boundary segregation on fraoture. Applying the newly developed theory, the group suggested a particular heat treatment to limit phosphorous segregation to the grain boundaries. While this treatment substantialiy contradicted accepted metallurgical practice it was tested and proved extremely successful, leading to several patents as well. Several important conclusions can be drawn: nology

- The theoretical model provided the interface with tech-

- The actual area of application of the new basic results (metallurgy) had nothing to do with the intended application (catalysis) 
$-9-$

- Channels of direct communication between basic and applied researchers were important. 
IV. INTRODUCTION TO PROBLEM AREAS

A. Selection Process

In understanding either the intrinsic properties of a surface system or its interaction with experimental probes, one is concerned with

- Atomic geometry

- Electron states and excitations

- vibrational excitations

- Chemical reactivity.

All of these aspects of the problem are tied together, and thire is no real justification for considering them separately. Nevertheless, theorists in the field tend to concentrate on one aspect. Just as an experimentalist builde apparatus and develops techniques focusing on one particular kind of measurement, a theorist develops approximations and calculational tools suited to studying some particular aspects of the behavior of a variety of surfaces. Relating the results of different kinds of studies on a given system is obviously important, but this is not where most of the effort is usually invested.

The panel sought to identify a number of important "problem areas," which might be defined as units of research activity which could occupy rost of the attention of an individual theorist or small group for a year or more. A list of over 50 topics was finally merged into 24 . Several different criteria were applied in the selection process, and weigh differently for. different problem areas:

- Recent activity and success

- specific present need in support of other research

- potential long-term importance.

The grouping of material into problem areas is of course to some extent arbitrary, and the panel certainly could not make strong arguments against combining some areas on the list or dividing others. There is, unavoidably, a certain amount of overlap. In most cases, the distinctions between theories of systems and theories of probes is maintained where appropriate. The distinction between first-principles and model theories is used to divide areas in the especially active electronic structure field.

No attempt was made to assign priority rankings to the problem areas. There is no single scale on which they could all 
be measured, and it was felt that no useful end would be served by a ranking.

The problem areas have been loosely grouped into three sections in organizing the report: areas focusing chiefly on electrons, areas focusing chiefly on atoms, and those involving both.

B. Format of the Problem Area Discussions

The chairman requested the panelists to adhere to a particular outline as much as possible in the individual writeups. While this necessitates certain redundancies in some cases, it puts various discussions on a more equal footing and aids the reader in scanning through those sections. The subsections of each write-up are:

1. Problem area.-- brief description of the issues involved if not clear from the name

2. Method of approach -- general nature of theories which have been or might be used

3. Long-term goals - what we hope the theoretical research will eventually accomplish

4. Impact on other theoretical areas

5. Impact on experimental areas

6. Impact on applied areas

7. Development to date

8. Anticipated future development -- reasonable extrapolations from 7 as distinct from 3

9. Needed supporting efforts -- experimental, theoretical in other areas, computational, etc. 
V. PROBLEM AREAS FOCUSING ON ATOMS

A. LEED STRUCTURE DETERMINATIONS

F. Jona, S. Y. Tong

1) Prublem Arca

- The determination of atomic arrdugemento on solid surfaces is an important, ullen ncoessary, prerequisite for the understanding of surface mechanisms and processes in a variety ur lechnologically important areas

- LEED (in the dynamical theory mode) has been shown to be a very sensitive technique for the determination of surface structures on the atomic scale

2) Method of Approach

- A LEED structure analysis consists usually of four steps: (i) preparation of the surface structure and collection of LEED intensity data: (ii) postulation of structural models consistent with the symmetry and the geometry: of the LEED pattern observed; (iii) calculation, with dynamical theory, of the elastic backscattered intensities expected from the postulated models: (iv) evaluation of the agreement between calculated and observed intensities

- There are no strict rules for either (ii) or (iv). The former is left to the inspiration and the experience of the worker. The latter has been carried out with the subjective, qualitative and imprecise method of visual judgment of the theory-experiment correspondence. Nevertheless, when a satisfactory and convincing fit is found between calculations and observations, the model is believed to be correct with a high degree of probability

- There may be systematic errors, and the estimates are uncertain, but most of the structure must be close to the postulated one -just because of the basic sensitivity of LEED to the geometry of the surface layers, and because the amount of information that is fitted with just a few adjustable parameters is very large

3) Long-Term Goals

- The long-term goal of LEED crystall cgraphy is to make the determination of surface structures involving one or more (up to five or six) atomic layers, a routine procedure easily accessible to the surface scientist, reliable, quantitative and relatively 
inexpensive even for complicated cases of multilayer adsorption or surface reconstruction

4) Impact on Other Theoretical Research

- LEED crystallography has already had tangible impact on other theoretical research. since the theoretical apparatus that was developed for the calculation of LEED intensities is being used extensively in the theories of angular-resolved photoemission. of electron-loss and of auger spectroscopies

- The knowledge of the atomic structure of solid surfaces is an essential input for calculations of surface electronic band structures, surface states, for the conception and evaluation of atomistic mechanisms of surface reconstruction, of surface reactivity. of catalytic phenorena, of crystal growth, of excitation and corrosion processes, etc.

5) Impact on Experimental Research

- The impact on experimental research consists perhaps in the stimulation of experiments : to test electronic structure and bonding schemes calculated or fostulated on the basis of the knowledge of the atomic structure

6) Impact on Applied Areas

- Understanding of surface reconstruction and chemisorption characteristics is likely to have impact on the growth of bulk or thin film semiconductors, epitaxial layers and passivation procedures

- Chemical reactivity. mechanisms of chemical reactions and surface bonding. all of which would ke better understood with the knowledge of atomic structure, represent the keys toward solintions of technologically vital problems such as catalysis. fuel research, energy production, corrosion phenomena and crystal growth

7) Development to Date

- The structural information that is available to date in surface science comes almost exclusively from LEED

- In the area of clean nonreconstructed surfaces, some 25 lowindex $(\{100\},\{110\},\{111\})$ surfaces of metals and elemental semiconductors, and 3 or 4 of binary compounds have been investigated. In the former group, the results concern primarily the existence and the magnitude of the top-layer relaxation. In the latter group, the most interesting results concern the reconstruction of the GaAs\{110\} surface, with the As atoms moving out and the Ga atoms moving into the surface 
- Mcst of the activity in the past few years has been in the area of ordered chemisorbed layers. where some 40 different structures have been analyzed. In most cases, adsorbed atoms have been found to reside on "expected" locations, i.e.. an the sites that would be occupied by atoms of the substrate if the latter did grow. However, several notable and interesting exceptions have been found, including "stacking faults," occupation of "bulk-interstitial" sites and ordered "underlayers."

8) Anticipated Future Development

- The computer programs used for the calculations of LEED intensities must be streamlined, made versatile and easy to use by the nontheorist in order to attract more wurkers (especially experimentalists) into the field

- Objective criteria for the evaluaticn of structural models must be developed or else those " $r$-factors" that have already been proposed must be improved and made available to all IEED workers. Sensitive r-factors are useful and important for two reasons: (i) they can and should be used for refining the structure under study and for evaluating quantitatively and objectively the credibility of proposed structure models; (ii) they may constitute the tool for the development of badly needed search procedures to guide the surface crystallographer away from wrong models in the direction of the right model (absolute minimum of the $r$-factor)

- New. efficient calculation schemes are needed for the study of high-index, or stepped surlaces, where the atomic interlayer spacings can become so small (cf the oraer of $0.5 \lambda$ or less) as to cause catastrophic numerical instabilities in the multiplescattering plane wave expansions commonly used in present-day computer programs. The quantitative study of stepped surfaces is long overdue for the understanding of chemical reactivity. passivation. inertness and poisoning that play important roles in catalytic and corrosion phenomena

- LEED structural studies of molecular adsorption, especially when the adsorbed molecule is known or expected to project from the surface into vacuum (e.g.. CO on $\mathrm{Ni}$ ) are plagued with the uncertainty about the scattering potential of the ndsorbed molecule. In the area of atomic adsorption, the great simplifying feature of : LEED for structure analysis is indeed that above some moderate energy of 30 to $40 \mathrm{eV}$ the effective potential is known and spherical. Surface theory should entertain and answer the corresponding question for the case cf molecular adsorption

9) Needed Supporting Efforts

- LEED crystallography can profit from, and in the future should be used in conjunction with, other experimental and theoretical structure-sensitive tools. Ion scattering, photoemission, atom scattering. ELS, and SEXAFS can provide invaluable information 
about the type of surface bonding. the occurrence or not of molecular dissociation, the orientation of molecular species relative to the surface, nearest-neighbor distance, etc.

- These techniques may provide independent sources of interatomic distances that are needed in order to pick up systematic errors in LEED analysis (such errors may stem from somewhat inaccurate potentials. neglect of parameters such as the second substrate interlayer spacings, etc.), and in order to establish the accuracy (as opposed to the precision) of LEED structure determinations

\section{Additional comments}

- At the present time, the number of groups doing LEED crystallography is dangerously small, and for this reason the field advances slowly and somewhat hesitantly. There is also the danger of absence in direct cross-checking

B. ATOM DIFFRACTION

J. D. Doll. M. Schluter

1) Problem Area

- The area of discussion here is that of atom/solid-surface diffractive scattering, and, in particular, the development of theoretical methods for the treatment thereof

2) Method of Approach

- A portion of this problem is nearly thenry free. surface resonance experiments (selective adsorption) provide an essentially direct measure of the bound states of the bulk-gas interaction (the gas/surface interaction averaged over the unit cell)

- The more involved problem of the calculation of the intensities of the various diffraction beams as a function of incilent energy has been addressed using a variety of quantum and semiclassical techniques.

3) Long-Term Goals

- Atom surface diffraction offers an important surface probe. Unlike electron or optical scattering experiments the energy range and the nature of the scattering assures a puxely surfacesensitive technique

- Both information conceming surface order (or its absence) and the gas/surface interaction potential are provided. The extrac- 
tion of the surface order information is straightforward. The information concerning the gas/surface potential is provided by the variation of the number and intensity of the diffraction spots as a function of beam energy

- The theoretical problem is thus analogous to that of LEED, namely the extraction of information by matching calculated intensity vs energy curves for various postulated potential models

- The connection between atomic geometry and atom potential models must be established to turn atom diffraction into a structural tool

4) Impact on Other Theoretical Research

- The collision theoretical techniques lnvolved in these studies are similar to those of gas-phase molecular collision problems. Advances in methodology in one area are thus applicable to the other. To date the flow has principally been from the gas-phase to the surface problem

5) Impact un Experimental Research

- The principal experimental area that will be impacted will be LEED. The presence of complementary information from the atom surface diffraction experiments will provide an important cross chcck: for LEED structure assignments

7) Development to Date

- Surface resonances: In the earliest work on selective adsorption Lennard-Jones and Devonshire assumed the process was one of diffraction into a bound state of the average gas/surface potential. The measured resonances wero then matched to the levels of a postulated potential (typically a Morse) and the potential parameters were extracted. Recently Leroy has developed methods to deduce more accurate long-range forms of these potentials. With the availability of more recent experimental data more work of this type is warranted

- Intensities: After the earliest work by Lennara-Jones and Devonshire [1] quantum mechaical efforts have followed either a perturbative treatment (CCGM theory) [2] or a more ambitious numerical approach. [3] This latter approach is, in principle, capable of extremely high accuracy. However, its applicability is somewhat limited since the number of open channels is rather high at typical energies

- Semiclassical approaches, which have been quite successful in gas-phase heavy particle scattering, have been extended to the atom surface diffraction. [4] These techniques appear to be rather promising in that they are relatively simple and yet quantitative 
- More recently, development of quantum mechanical solutions for the hard-wall potential have been presented by Garabaldi and Garcia (see also R. I. Masel, et al [5])

- The extent to which any of the above methods provides a suitable tool for the analysis of atom/surface diffraction experiments is as yet unclear. The availability of data has, until recently, been rather limited. With the new generation of velocity analyzed data now available a clearer assessment of the merits of the above methods is possible

8) Anticipated Future Development

- The inclusion of lattice motion has essentially been ignored in the theoretical treatments of diffraction to date. Only in a primitive way has this point been addressed

- The effect of inelastic processes on diffraction must be understood and included.

- It should be noted that the standard Debye-waller treatment of thermal smearing is suspect here due to the comparable time scales for gas and lattice motion. Furthermore. since surface phonon effects are likely to be observable, more work on this topic is needed

9) Needed Supporting Efforts

- The single most important need is for experimental data particularly for surface resonances, intensity vs energy measurements, and for surface temperature variations

- Input on atom-surface potentials from electronic structure calculations would be valuable

\section{REFERENCES}

1. Lennard-Jones and Devonshire, Proc. Boy. Soc. A158, 253 (1937).

2. N. Cabrera, V. Celli, F. O. Goodman, R. Manson, Surf. Sci. 19, $67(1970)$.

3. G. Wolken, Jr... J. Chem. Phys. 58, 3047 (1973): 59, 1159 (1973).

4. J. Doll, J. Chem. Phys. 3, 257 (1974); J. Chem. Phys. 61. 954 (1974): D. R. Dion and J. Doll, Surf. Sci. 5ㅇ․ 415 (1976); R. I. Masel, R. P. Merrill and W. H. Miller, Surf. Sci. 46, 681 (1974); J. Chem. Phys. 6u․ 45 (1976).

5. R. I. Masel, R. P. Merrili and w. H. Miller, Phys. Rev. Bli2, 5545 (1975). 
C. SURFACE VIBRATIONS: INTRINSIC PROPERTIES AND EXPERIMENTAL PROBES

D. Mills, J. Joannopoulos, W. Plummer

1) Problem Area

- The theoretical problem is the calculation of surface phonons (or vibrational modes) for a wide variety of surface conditions and to couple these calculations into experimental techniques by calculating the response of the surface phonons to experimental probes

- The phonon modes of a surface are directly related to the interatomic force constants of the surface atoms. Most of the key issues in surface srience today are directly related to understanding the interatomic forces

2) Method of Approach

- Bulk lattice dynamics methods can be adopted for the solution of the vibrational modes of ordered single crystal surfaces

- Force constants and dynamic effective charges entering into models are presently determined empirically

- Methods for dealing with less symmetric surfaces, for treating most aspects of coupling to experimental probes, and for predicting model parameters remain to be devised

3) Long-Term Goals

- It should be possible to reliably interpret experimental data from probes that couple to vibrational modcs in terms of force constants alld dynamic charges

- Changes in force constants at the surface from bulk values in the case of substrate atoms or from molecular values in the case of adsorbed species should be understond in terms of electronic structurc or other " basic theoretical cönsiderations. Systematics of force constant variation as a function of bonding geometry and chemical parameters, established from either basic or empirical models. would be extremely valuable

- The role of surface vibrations in reconstruction, adsorbed atom interactions, and a variety of kinetic phenomena should be understood

4) Impact on Other Theoretical Research

- Knowledge of vibrational modes and their coupling to atomic and molecular probes is important in developing theories of accommodation and other surface kinetic phenomena 
- Adsorbate frequency shifts, mode dispersion, and dynamic effective charge provide further stimulus for electronic structure calculations

- Softening of surface phonon modes may be of importance in the theory of reconstruction

5) Impact on Experimental Areas

- Experimental techniques like surface EXaFs, high energy ion scattering, atomic scattering, LEED and ultraviolet photoelectron spectrcscopy require knowledge of surface vibrations in order to extract geometrical structure information reliably. In several experiments, correlated atomic motions may flay a crucial role, and proper account of these requires the theory to go beyond intrcduction of Debye-waller factors into a single site $t$ matrix

- Experiments that attempt to measure the phonon spectrum directly include high resolution inelastic electron scattering, and optical probes such as infrared and Raman spectroscopies. In these cases, a major theoretical effort is required to interpret the experimental data

6) Impact on Applied areas

- The weakening of molecular bond force constants upon absorption plays a major role in dissociation and hence catalytic reactions

- Modification of substrate force constants by chemisorbed species results in effects like corrosion embrittlement

7) Development to Date

- For ordered crystals, i.e.. clean surfaces of single crystals, possibly with an adsorbed overlayer in registry with the substrate, a variety of methods exist for calculating the dispersion relation for surface phonons, the mean-square displacement of surface atoms and other features of the surface vibrational motion. This has been done for models of surfaces of metals, semiconductors and ionic crystals, though semiconductors have received less attention

- Quantitative results require knowledge of the atomic force constants and the dynamic effective charges for the surface atoms

- In a number of studies, information about force constants comes from contact with surface mean-square displacement data extracted from LEED. The surface dynamic effective charge, if appreciable or greatly different from that in the bulk, has been shown to influence surface lattice dynamics importantly, though it has received little attention 
- Very little work has been done on the vibrations of adsorbed atoms or molecules, for models which incorporate the substrate motion in the vicinity of the adsorkate

- There is no body of theory that addresses surfaces with a disordered adsorbate layer present at finite coverage

- Simple models of surface vibrations on amorphous materials have been developed and brought into contact with data, but this remains a tckic that has received limited treatment

- Spin waves at magnetic surfaces may be studied by methods similar to those used in surface lattice dynamics. An extensive theoretical literature exists on theory of maynetic sirface phenomenon, including surface spin instabilities. Lifele is known about exchange constants at surfaces, and few experiments exist that lead to quantitative contact with theory, tlough recent light scattering studies look encouraging

- The coupling of surface vibrations to low energy electrons inelastically scattered near the specular direction or near a Bragg peak is dominated by dipolar coupling and is well understood

8) Anticipated Future Development

- Recent experimental work explures large angle inclastic scattering from adsorbates on metals. There is as yet no theory of the large angle scattering, outside the intense near specular scattering dominaten hy dipolar coupling to the adsorbate vibratinn. We have no understanding of the physical origin of the resonances cbserved in the large angle loss cross sections. The methods of IEED theory must be applied to address this regime. A considerable theoretical effort should be devoted to this in the near future

- Recent Raman studies of molecular vibrations at the interface between electrolytes and notle metals find cross sections 1,000,000 times larger than gas-phase Raman cross sections. There is no theory of the origin of this huge, nonresonant enhancement; a successful thenry may lead to fundamental new insights into the coupling between electronic states of the substrate and the adsorbate 
D. INTERACTION OF MOLECULAR BEAMS WITH SURFACES

J. D. Doll, T. Fischer

1). Problem Area

- Interpretation of molecular team scattering, adsorptiondesorption, and surface reaction experiments

- Given a molecule-surface potential, perform the collision dynamics treating, among other things, energy loss or accommodation, sticking, reaction kinetics, and surface nucleation phenomena

- The determination of the molecule-surface potential

2) Method of Approach

- For the treatment of the dynamics of these complex systems the predominant technique is classical collision theory

- Incorporation of modern nonequilibrium statistical mechanical methods has proven convenient

- In some instances (e.g.. accommodation) quantum mechanical collision rethods are available. At present empirical potentials are used

- Input from electronic structure theory is badly needed

3) Long-Term Goals

- The long-term goal of this work is a theory capable of treating the dynamics of surface processes (both inelastic and reactive)

- An important goal is the ability to understand, even in a primitive way, the substrate:s modification of reaction dynamics

- Another important area concerns prccesses in which adsorbate modifications of the substrate occur (e.g.. chemisorptive embrittlement)

4) Impact on other Theoretical Research

- The ability to study the dynamics of many-particle systems is one common need in several areas of investigation (e.g.. nucleation phenomena and gas-phase collision theory)

5) Impact on Experimental Research

- The ability to interpret increasingly complex surface processes will (hopefully) encourage increasingly refined experimental studies 
- The need for high resolution, velocity- selected (initial and final) atom/surface scattering experiments is evident

- In analogy with gas-phase developments, experiments studying the effect of internal (molecular) energy would be useful

- In materials science, the effects of any advances in the problem of adsorptive modification of lccal cohesion would be appreciable

6). Impact on applied areas

- The predictive power of the theory of surface bonding and reactivity will, if realized, contribute importantly to a number of applied areas. For example, the description of the modification of substrate cohesion by adsorption would lead to important materials progress, particularly in embrittlement phenomena, lubrication, adhesion, and corrosion

7) Development to Date

- The electronic structure aspects of the present problem are described in other sections

- The collision dynamics of these processes must, of necessity, be capable of treating large numbers of degrees of freedom. Furthermore, averaging over the lattice degrees of freedom is always present. Consequently a classical collision theory is suggested

- A review of past theories 15 given by Weinberg [1]

- More recently attempts have been made to simplify the collision dynamics by including the effects of the majority of them within a generalized Langevin equation framework [2]

- Probleils involving surtace recombination and dissociation have been addressed by wolken, et al, and by Cardilio and Gelit; réspectively

8) Anticipated Future Development

- The most important future development will be the inclusion of the electronic degrees of freedom in the above scheme. Progress in this direction has been made by $w$. Schaich

9) Needed supporting Ef forts

- Experimental support, especially in the form of high resolution molecular beam surface scattering experiments, is certainly needed.

- Input for the present work involves quantities determined by lattice dynamical and electronic structure studies 
REFERENCES

1. H. Weinberg, Adv. Colloid and Interface Sci. 4.301 (1975).

2. S. A. Adelman and J. D. Doll, Accts. Chem. Res. 10. 378 (1977); R. Zwanzig; J. Chem. Phys. 32 ; 1173 (1960); F. O. Goodman, J. Phys. Chem. Solids 23. 1269, 1491. (1962).

E. REACTION KINETICS OVER SURFACES

H. Suhl, J Doll, P. P. Schmidt

1) Problem Area

- Expressing the quantities entering rate constants in terms of substrate properties, such as respcnse functions, thermodynamic quantities, critical properties near phase transitions

- A particularly poorly understood area is that of reactions over insulators, because of the equality of time scales of substrate and reactant motions

2) Method of approach

- statistical mechanics and thermodynamics

- For the case of insulators, a quasi-particle approach (reactants "dressed" with virtual phonons)

- Simple inequalities for extreme cases

3) Long-Term Goals

- Relating catalytic processes to solid-state physics

- Trying to abstract from a detailed study of a sizable number of significant special cases some general principles that can be used under "field" conditions (real catalysts, etc.)

11) Impact on Other Theoretical Research

- Kinetics in general

- Nonequilibrium thermodynamics

- Actually kinetic theory at this level probably will borrow more than it will give

5) Impact on Experimental Research

- clarification of some experimental puzzles, e.g.. compensation etfect in catalysis 
- Fluctuation spectroscopy of reaction rates

- Guidance for critical experiments

6) Impact on Applied areas

- Eventually, design of catalysts with prescribed characteristics

- corrosion

- Afplication of oscillatory reactions to produce anomalously high or low average reaction rates, or anomalous catalyst specificity

7) Development to Date

- General rate theory formulated by kramers [1] is being reexamined and extended using modern many-body theory [2]

- There is a considerable amount of new theoretical work on kinetics in general. The Kramers model does not offer the flexibility needed for these types of analyses. Correlation function approaches must calculate the rate constant directly rather than the friction constant in the kramers theory

- A few detailed calculations of phonon-assisted desorption have been carried out $[3,4]$

- The role of substrate magnetic fluctuations in surface rates, especially near phase transitions, has been examined $[5,6,7]$

8) Anticipated Future Development

- Synthesis of static results (calculation of energy surfaces) with kinetic calculations

- Understanding of magnetocatalytic effects

- Experimentation on, and analysis of time-dependent scattering, photoemission, etc.. measurements tc"probe the spectrum of timeconstants of surface reactions

- Determination of adiabatic (Born-oppenheimer sense) stationary states

- Relations between the dissipative and reactive effects of adatom coupling to substrate fluctuations could be derived and exploited.

- Irreversible thermodynamics of surface reactions.including onsager coupling coefficients of the reaction to the surface transport properties should be, studied 


\section{REFERENCES}

1. H. A. Kramers, Physica 7, 284 (1940).

2. E. G. d'Agliano, P. Kumar, W. Schaich and H. Suhl, Phys. Rev. B 11. $2122(1975)$.

3. G Iche and P. Nozieres, J. de Physique 37. 1313(1976).

4. B. Bendow and S. C. Ying, Phys. Rev. B I. 622 (1973) and I. 637 (1973) . (ca. 1972).

5. H. Suh1, Rhys. Rev. B 15, 5657 (1977).

6. P. Kumar, J. H. Smith and H. Suhl, Phys. Rev. Lett. $2 \underline{5}, 1442$ (1970)

7. E. Ilisca, Phys. Bev. Lett. 40. 1535 (1978).

F. INTERFACE TRANSPORT PROPERTIES

P. Schmidt, J. Doll, R. Messmer

1) Problem Area

- Transport phenomena is understood here to encompass diffusion to the surface through the interfacial double layer, lateral diffusion in the interface, and cherical and electrochemical reactivity

2) Method of Approach

- There are now established theoretical approaches to the description of electrochemical charge transfer. These methods essentially apply the solid-state polaron theory to the reactive species in the interface. Care is taken to account for the particular chemical effects which differ from the solid-state polaruil system

- The irreversible thermodynamics of material and thermal transport in the interface is not developed

3) Long-Term Goals

- An electrochemically produced electric current of a given potential depends predominantly upon mass transport processes. This is reasonably well understood. In a number of interesting cases there is a substantial dependence of the current upon the charge transfer act

- The general theory of electrocherical reactions needs to receive continued support. The goal is to know enough about the reaction systems to be able to systematize and codify the experimental data

- It is probably unrealistic to expect that theoretical calculations will be able to give numerical agreement with experimental 
data. However, it is anticipated that theoretical calculations on separate systems should show trends or the absence of trends

- The irreversible thermodynamic study of matter transport in the interface should be encouraged. These processes are the analogues of defect and impurity migration problems in the crystal surface

- There is much important chemistry which is surface, or perhaps interface catalyzed. An ability to manipulate these transport equations is necessary to be able to handle the reaction kinetics

4) Impact on other Theoretical Research

- 3urface transport studies are not anticipated to have a large impact upon other areas of theoretical investigation. These transport problems generally require the appliceation of theoretical methods developed for a variety of problems in order to obtain a solution

5) Impact on Experimental Research

- The experimental impact is very great. Reactivity and transport phenomena can often be tested relatively easily. More important, however, is the possibility offered by the theoretical unification of apparently diverse phenomena.

- The knowledge that systems of reactions and interfacial processes can be grouped according to similar qeneral properties will greatly facilitate further experimentation

- Theoretical work in this area can be a labor-caving device to the experimentalist

6) Impact on applied Research

- Benefits from theoretical development similiar to those ill section 5 above chould aid areas important in energy technology

7) Development to Date

- Simple electrochemical reactions are reasonably well characterized

- The role of surface states has not been investigated. However. it has been found that metalic band structure is relatively unimportant for the most part. The simplest metalilc models have been used and work reasọnably well

- Semiconductor valence and conduction bands contribute independently. Both must be considered in evaluating the electrochemical current. Nevertheless, in spite of the great differences between metals and semiconductors, the experimental 
results in the two cases look somewhat similar from the point of view of a coarse-grained experiment, viz. a simple current vs potential measurement. On the other hand, combined optical and electrochemical experimental studies do show a considerabie complexity, particularly. in semiconductor systems.

- There has been some effort -- especially recently -- to develop other methods to probe the interfacial state. The activation energy for an electron transfer transition is now about the only quantity which can be examined theoretically with any hope of experimental agreement. This work continues

- New data from other types of measurements are being incorporated into the analyses

- The irreversible thermodynamics is a relatively unexplored area to date

8) Anticipated Future Development

- One of the important problems which needs to be tackled is the question of "chemical adiabaticity" with reference to reactivity. The present formalism depends upon the use of first and second order time-dependent perturbation theory. Thus, reaction rate constants derived on this basis are bound to the nonadiabatic limit. This aprearas not to be a grave difféiculty for boulk solution electron transfer. Those reactions seem to be slow enough to admit the nonadiatatic limit. Electrode reactions, on the other hand, seem to demand the adiabatic limit. work in this area is needed

9) Needed Supporting Efforts

- One of the major roadblocks to the rapid development of electron transfer theory has been the fact that far too few people have worked on it. This is changing slowly

- Specific encouragement of research in the electrochemical area is needed. Both theoretical physicists and chemists should te encouraged. The history of research in this area has been equally dominated by theoretical chemists and physicists 
G. ADSOBBATE-ADSORBATE INTERACTIONS

W. Plummer, D. Mills

1) Problem Area

- To understand the physics cf transverse interactions between adsorbates on a surface, and their consequences both in physisorbed and chemisorbed systems (i.e... a continuous range from weak to very strong bonding)

- Indirect mechanisms mediated by the substrate need further study

2) Method of approach

- A variety of theoretical approaches can be envisioned, depending on the situation. In one extreme (chemisorption and two adsorbates on nearby sites), one has a problem suitable for analysis by cluster methods, or other modern approaches to the electronic structure of surfaces

- In the physisorbed limit, perturbation theory may be used to explore various couplings

- Once couplings are modelled, in both cases one is led to fundamental statistical problems

3) Long-Term Goals

- To understand the short-range and $1 \mathrm{cng}$-range order that occurs in adsorbed layers. One has order-disorder transitions in both systems, diffusion of small complexes (pairs and triplets) in chemisorbed systems: these can be treated by statistical methods. given the transverse couplings

- The physics of the diffusion and desorption processes shoula be understood

4) Impact on Other Theoretical Research

- These issues are intimately related to fundamental issues in the kinetics of reactions on surfaces

- Theories of chemical bonding on surfaces can provide, with appropriate focus, information of value here

- Fundamental theoretical issues concerning the nature of phase transitions in two dimensions and commensurate-incommensurate transitions may be tested in the context of this problem area

5) Impact on Experimental Research

- There are presently many studies of short-range oraer, longrange order, and phase transitions in these systems 
- Diffusion and desorption have been studied, but at present the theory is far too primitive to account for the systematic features and trends present

- Existence of a body of theory may reveal important relationships unsuspected at present

6) Impact on Applied Areas

- Many cases are known where less than one monolayer of material (S. on Pt(100), for example) completely poisons an otherwise active catalyst.[1] The advances in understanding required to address the issues just outlined may provide insight into this phenomenon

- These issues are intimately related to surface reaction kinetics and crystal growth mechanisms

7) Development to Date

- sophisticated models of order-disorder transitions in physisorbed systems have been developed to a high level, but the transverse interactions that enter are phenomenological, poorly understood adjustable parameters

- Little attention has been devoted to chemisorbed systems

8) Anticipated Future Development

- Scme progress along the lines indicated in sections 2 and 3 may be anticipated

- Significant progress will probably involve new approaches

9) Needed supporting ifforts

- Fundamental theoretical studies of the electronic structure of surfaces in systems with adsorbates

- More information on surface lattice vibrations and electronphonon coupling at surfaces, to evaluate indirect interactions through phorions

\section{REFERENCES}

1. T. E. Fischer and S. R, Kelemen, J Catal. 므. 24.(1978). 
H. PRECURSORS

T. E. Fischer, D. R. Hamann, W. E. Spicer

1) Problem Area

- Gases can adsorb on surfaces in precursor states from which they may either desorb, chemisorb, or react with a second species. such states may be extremely important in. for example. catalytic reactions, but are very hard or impossible to directly detect and study because of their transient nature

- Theoretical questions have to do with the dynamics of the loss of kinetic energy of the impinging gas and with the real potential defined by the gas and substrate

2) Method of Approach

- More experimental information must be gathered to guide theory to the proper concepts

- Loss processes involving the gas and surface are important. Knowledge of phonon modes may be critical here

- The precursor state must be described in sufficient detail so that reaction probability with chemisorbed, or other, species can be understood

- The dynamics of desorption of the precursor must be worked out theoretically

- It is possible that the conventional reaction-coordinate picture is misleading here and needs to be replaced by a more sophisticated concept

3) Long-Term Goals

- Theoretical description of the precursor state

- Identification of the phenomena and properties resfonsible for its existence and its detailed role in, for example, specific catalytic reactions

4) Impact on other Theoretical Reșearch

- Solution of this problem is demanding enough to stimulate other related research, e.g, on phonon modes

- It is likely to generate new concepts or insights into the theory of chemisorption and surface reactions 
5) Impact on Experimental Research

- Collaboration between theory and research will accelerate progress in this area

- Lack of knowledge of the precursor states may be a missing link between present experiments and the understanding of surface chemical phenomena

6) Impact on applied areas

- Impact is probable on catalysis and other surface-chemistry phenomena, especially through progress in general understanding of chemisorption and surface reactions that will be stimulated by work on this problem

7) Development to Date

- Any descriptions to date of the precursor state are in terms of simple. ad hoc qualitative potentials that do not provide sufficient insight into this problem

8) Anticipated Future Development

- Difficult to predict as the basic concepts and hypotheses on this phenomenon are not presently available; however, it should lead to better understanding of the potential surfaces defining reaction paths

9) Needed supporting Efforts

- close two-way information exchange with experiment is essential. The question of whether or not steps or other defects play an important role with precursors is very important in theoretical considerations

- Theorists, in general, do not seem to be sufficiently aware of this problem: Increasing awareness, particularly by theorists working in adjacent fields such as that of binding energies of physi- and chemisorbed species, would undoubtedly allow them to also contribute to this field with little extra effort 
I. RECONSTRUCTION MECHANISMS

D. R. Hamann, M. Schluter, F. Jona

1) Problem area

- Many semiconductors and some metals have surface superlattice structure

- Geometrical structure and driving forces responsible for structure are unknown in most cases

- Phase transitions among different structures are common, indicating small energy differences are involved

2) Method of Approach

- To understand structure, LEED intensity analysis and electronic structure calculations for simple cases

- To understand driving forces, instacility analysis of unreconstructed surface involving electronic energies, lattice force models, electrostatic forces, or a combination

- Simplified models extracting key elements undoubteāly necessary for complex cases

3) Long-Term Goal.s

- It is probable that there is not a single reconstruction mechanism, but several that apply in the cases of different systems. It should ultimately be possible to classity the dominant reconstruction mechanisms for various surfaces from a knowledge of bulk properties.

- The effect of reconstruction on the chemisorption properties of a surface. and in turn, the effect of adsorbates on the reconstruction must be understood.

- Models capable of. predicting the temperature dependence of reconstructed phases should be developed

- changes in reconstruction produced by electronic excitations should be possible and should be explored and understood

4) Impact on Other Theoretical Research

- Ordering in aảsorbed layers probably has common features with reconstruction, and may use developments here

- Theoretical treatment of crystal growth deals with related questions 
- Charge density waves in layered materials involve related effects

- Jahn-Teller distortions around defects and voids in amorchous materials are related

5) Impact on Experimental Research

- There is a large body of data on the effects of reconstruction on surface spectra, work functions, and Fermi level pinning, most of which is unexplained

- Surface phonons and their coupling to experimental probes are strongly effected by reconstruction

- If connections are established between some reconstruction mechanisms and párallel bulk effects. application of some bulk experimental techniques to surfaces may be stimulated

6) Impact on Applied A reas

- Epitaxial growth of semiconductor devices is strongly influenced by reconstruction

- Reconstruction undoubtedly influences the catalytic properties of surfaces

7) Development to Date

- Existence of reconstruction known for 20 years

- Most early work consisted of postulating geometries and mechanisms such as rebonding, rehybridization, ordering of surface vacancies, and charge density wave distortions

- For several simple cases on Si surfaces, electronic structure analysis has given strong evidence that particular models are correct $[1,2]$. Evidence from LEED analysis is not yet convincing for any system

- Instability analysis carried out on the basis of simple models involving charge density waves or soft phonon modes has been discussed for a few cases [3,4], but this development is in its infancy

8) Anticipated Developments

- Further LEED analysis and refinements of models will probably produce an accurate knowledge of the geometry of a number of simple reconstructed surfaces within the next few years

- Electronic structure should be studied, especially for surfaces where LEED determinations have been completed 
- Adequate tools are available to study the vibrational properties of reconstructed surfaces

- A start could certainly be made toward modeling the energetics of. the simpler surfaces that undergo phase transitions

- Combined theoretical and experimental efforts to determine whether or not large concentrations of vacancies exist on any surface are necessary, and the results will. strongly influence further development of models

9) Needed Supporting Efforts

- Primarily experimental data; development of theory should indicate key experimental issues

- Low-temperature experiments may reveal hitherto unknown new reconstructed phases.

REFERENCES

1. J. A. Appelbaum and D. R. Hamann, Phys. Rev. Lett. 35,729 (1975): Phys. Rev. B14. 588 (1976); Phys. Bev. B 1 15. 2408 (1977) .

2. J. A. Appelbaum and D. R. Hamann, Phys. Rev. B12, 1410 (1975); K. C. Pandey and J. C. Phillips, Phys. Rev. Lett. 34.41450 (1975): M. Schluter, J. R. Chelikowsky, S. G. Louie, and M. I. Cohen, Phys. Rev. Lett. 3 $3 \underline{4}, 1385$ (1975).

3. E. Tosatti and P. W. Anderson, Solid state Comm. 14. 773 (1974); J. A. Appelbaum, G. A. Baraff and D. R. Hamann, phys. Rev. Let.t. 36. $450(1976)$.

4. S.E. Trullinger and S. L. Cunningham, Phys. Rev. Bי, 2622 (1973). 
VI. PBOBLEM AREAS FOCUSING ON ELECTRONS

A. SEMICONDUCTOR AND SIMPLE METAL SURFACE ELECTRONIC STRUCTURE -PSEUDOPOTENTIAL CALCULATIONS

M. Schluter, J. D. Joannopoulos

1) Problem area

- Calculations of the electronic structure of surface systems, including:

- clean surfaces and reconstructions

- surface defects and chemisorption

- heterojunctions and schottky barriers

2) Method of Approach

- The electronic structure of surface and interface systems is calculated based on self-consistent pseudopotential schemes. These include the use of slabs and semi-infinite geometries to describe surface configurations. The results are of a quantitative nature

3) Long-'Term Goals

- The understanding of chemical bonding at surface and interface systems

- The calculation of total energies and its application to determine surface structure, distortions and vibrational modes

- The calculation of experimental response functions

- Improvement of present techniques to make them more firstprinciples in character, and to make them applicable to more structurally complicated systers and materials

- Extension of the one-electron treatment to include localized. correlated electrons

4) Impact on other Theoretical Research

- Results serve as guidelines for simple empirical calculations. yield structural information as related to LEED theory. photoemission theory. SEXAFs theory. etc.

- The approach developed for treating surface systems has been used to improve calculations on bulk solids, molecules, defects; stacking faults and other localized systems 
5) Impact on Experimental Research

- The calculations yield quantitative spectroscopic results in direct comparison to surface spectroscopy measurements like ups. XPS. ELS, etc.

6) Impact on Applied Areas

- Understanding of several aspects of heterojunction lasers ana Mos devices

7) nevelopment to Date

- Reliable ionic pseudopotentials have been derived

- Methods to treat systems without complete periodicity have evolved and include boundary matching techniques aná supercell geometries

- Extension of standard plane wave basis set to include localized orbitals

- Realistic studies have been carried cut for: simple metal surfaces, such as $\mathrm{Li}$, Na and Al; semiconductor surfaces such as $\mathrm{Si}(111)$, Si (100), Ge(111), C(111), GaAs(110), ZnSe(110), and various reconstructions such as $\operatorname{Si}(111) 2 \times 1, \quad S i(100) 2 \times 1$, and steps on Si(111): chemisorption systems such as $\mathrm{Si}(111)-\mathrm{H}$, Si(111)-SiH3, Si(100)-H, Si(100)-H2, Si(111)-Cl, Si(111)-AI. GaAs(110)-AI; semiconductor heterojunctions such as GaAs-Ge, GaAs-AlAs, Znse-Ge: Schottky barrier studies. metal- (jellium) semiconductor junctions, including $S i, C$, GaAs and znse

8) Anticipated Future Development

- Extension to the study of transition metal surfaces, defects on surfaces, more complex reconstructicns, more complex chemisorbed species, calculation of total surface energies.o nore realistic metal-sericonductor interface structures

9) Needed Supporting Efforts

- Excellent computational facilities

- Development of formalisms to treat localized electronic correlations

- Better atomic (and molecular) pseudopotentials

- Better structural data from experiments 
B. SEMICONDUCTOR SURFACE ELECTRONIC STRUCTURE -TIONS

EMPIRICAL CALCULA-

J. D. Joannopoulós, M.: Schluter

1) Problem area

- Calculations of the electronic structure of ordered semiconductor surface systems including reconstructions, defects, chemisorbed species and interfaces between surfaces

- The calculation of the electronic structure at disordered surfaces and interfaces

2) Method of Approach

- The electronic structure of surface and interface systems is calculated using wave functions expanded in localized orbitals

- The Hamiltonian matrix elements may be calculated or treated as empirical parameters

- The surface:system can be modelled as a slab, semi-infinite solid, isolated cluster or a cluster embedded in an effective medium

3) Long-Term Goals

- The understanding of chemical bonding and nature of electronic states at surface and interface systems from a real space point of view

- Understanding the electronic structure of disordered surfaces and alloys

- The establishment of trends in the kehavior of clean and contaminated surfaces

- Development of optimized schemes, sophisticated enough to give quantitative information yet simple enough to be versatile for many calculations

- The calculation of total energies from empirical models

- Extension of one-electron approach to include localized correlated electrons

- Adequate treatment of excited states so that one can calculate response functions

4) Impact on Other Theoretical Research

- Results from empirical tight-binding calculations help interpret and understand more complicated first-principles like calculations 
- Yield structural information complementary to LEED, photoemission and SEXAFS studies

- Methods developed for surface studies can be used to imporve tight-binding bulk band structure calculation of crystals and electronic structure calculations of amorphous solids (e.g.. hy" drogenation of amorphous $\mathrm{Si}$ )

5) Impact on Experimental Research

- Calculations can yield results quantitative enough to be compared to cxperimental specliuscupic data. ror example, seemingly ccntradictory experimental measurements on oxidation

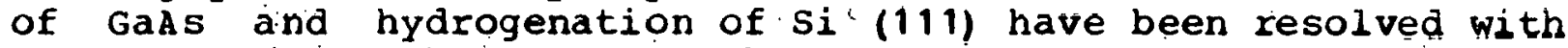
this localized orbital approach

- Trends in Schottky barrier formation have been established and may be of use in further experimental studies

6) Impact on Applied Areas

- Detailed studies of various bonding configurations at Mos interfaces have yielded the first quantitative theoretical information in this area and reveal the imfortance of surface related features

- Understanding of several aspects of heterojunction lasers

7) Development to Date

- Reliable techniques have been developed to derive empirical tight-binding parameters

- Empirical tight-binding studies have been used with slabs, semiinfinite and cluster-Bethe-lattice geometries to study $S i(111)$. $\mathrm{Si}(110)$. Si (100), Ge(111), Ge(110), C(111). SiC(110), GaAs(110). GaAs(111), - znSe (110) surfaces and scme of the major recorstruct.ions

- Chemisorption systems such as $H, C l$ and $O$ on $S i(111), C l, O$ and 02 on GaAs (1.10) have been studied

- Interfaces like GaAs-AIAs and Si-SiO2 have also been studied

- schottky barrier trends have been interpreted in terms of simple empirical models

- An ad hoc method of including effects of localized correlated electronic states for chemisorption studies has been introduced

- Finally a simple empirical technique for the calculation of total surface energies has been proposed 
8) inticipated Future Development

- Gain of quantitative character withcut the loss of simplicity. This would include increasing the basis set of empirical calculations

- Development of reliable schemes to adjust. surface atom parameters as geometry is varied

9) Needed Supporting Efforts

- Excellent computational facilities

- Experimental data for fitting studies

- Development of formalisms to treat localized electronic calculations

- Algorithms for fast matrix element calculations

C. TRANSITION-METAL SURFACES -- FIEST PRINCIPLES CALCULATIONS

L. M. Falicov, L. Kleinman

1) Problem area

- First-principles calculations of transition-metal electronic structure

- clean surfaces, chemisorbed layers and compound surfaces

2) Method of Approach

- Self-consistent slab calculations with correlations based on density-functional formalism

- Gaussian local orbital. linear augmented plane wave, or linear muffin-tin orbital wave function basis with full potential

- plane wave or mixed-basis wave functions with nonlocal pseudopotential

- Relativistic extensions are necessary for $5 d$ metals

3) Long-Term Goals

- Determine energy spectra, charge densities and wave functions in a large number of systems as a starting point to understand experimental data (e.g.. photoemission) and predict chemical trends and properties 
4) Impact on Other Theoretical Research

- strong ties and cross-stimulation with:

- many-body effects

- lccalized vs extended state controversy

- magnons at the surface

- surface energies and cohesion

- parametrized empirical methods

5) Impact on Experimental Research

- It should help in understanding:

- photoemission experiments

- chemi sorption data

- photochemical reactions

- ellipsometry

6) Impact on Applied Areas

- It may influence the understanding of corrosion and catalysis

7) Development to Date

- Several pseudopotential calculations for $4 \mathrm{~d}$ metals have been performed $[1,2]$. Convergence of flane wave basis is adequate because $4 \mathrm{~d}$ orbitals are not too localized. This rethod has not been develoced for 3d metals because of convergence problems associated. with highly localized 3d orbitals

- Full potential calculations have begun to be applied to 30 metals using localized orbital and linear APW bases [3]

- There have been several nonself-consistent first-principles calculations for transition and noble metals (e.g.. Fe, Cu) which are not very satisfactory because the potential is extremely sensitive to surface charge $[4,5]$

8): Anticipated Future Development

- Large activity is expected in all subfields in the near future. It will prohnhly be ons of the most active areas of surface science theory

9) Needed Supporting Efforts

- Large computational facilities are a must

Additional comments

- Chemisorbed layers present no additional basic problems if the atom positions are known.

- Transition-metal compounds may have the same serious problems they have in the bulk, i.e.. the kreakdown of the one-electron 
approximation due to correlation effects in narrow bands (Mott insulators)

\section{REFERENCES}

1. S. G. Louie, K. M. Ho, J. R. Chelikowsky, and M. L. Cohen, Phys. Rev. B $15.5627(1977)$

2. G. P. Kerker, K. M. Ho, and M. I. Cohen, Phys. Rev. Lett. $4 \underline{0}$ 1593(1978).

3. J. A. Appelbaum and D. R. Hamann, Solid state Comm. 227. 881 (1978).

4. E. Caruthers, D. G. Dempsey and L. Kleinman, Phys. Rev. B $1 \underline{4}, 288$ (1976).

5. R. V. Kasowski. Phys. Rev. Lett. $\underline{3} 3.83$ (1974): $3 \underline{3} \underline{3}, 1147$ (1974); 37. 219 (1976): Solid state Comm. 17. 179 (1975): Phys. Rev. B1ㅗ. $3398(1976)$.

D. TRANSITION-METAL SURFACES -- EMPIBICAL CALCULATIONS

L. M. Falicov, L. Kleinman

1) Problem Area

- Empirical tight binding

- clean surfaces and transition-metal compound surfaces

2) Method of approach

- Fitting of electronic bands by means of slater-Koster LCAO Hamiltonian coefficients to handle a variety of compositional and geometrical situations

- Surface self-consistency problems handled by ad hoc procedures

- Flexibility of choice of basis functions with addition of overlap parameters

3) Long-Term Goals

- Parametrization and description of experimentally determined bands. including many-body and spin-dependent effects

- Interpolation scheme for calculaticn of a large number of physical quantities based on a few carefully calculated firstprinciples quantities 
4) Impact on Other Theoretical Research

- It simplifies ways of handling many-body local (atomic) effects

- It permits easy handling of itinerant magnetism effects

- It can be directly connected to cluster and chemical approaches

5) Impact on Experimental Research

- It has already influenced research on (a) spin polarized emission from ferromegnetic metals, (b) photoemission spectroscopy (surface states)

- It may in principle have impact on any experiment related to the electronic structure of surfaces

- It has the capabilities of becoming the scheme for expressing directly the experimental results

6) Impact on Applied Areas

- By simplifying the theoretical language it may act as a direct tool for the transfer of basic information into applied areas

7) Development to Date

- Sophisticated schemes for reducing the large number of surface parameters to a significant few have been accomplished [1,2]

- Spin-polarized calculations for transition-metal ferromagnets have been completed. Spin-polarized photoemission experiments have been explained [2,3]

- Some very simple model alloys have been tackled by a variation of this method [4]

8) Anticipated Future Development

- High Miller index and corrugated surfaces [5] are in the process of being studied by this method

- Relativistic calculations for heavy (5d) transition metal will be forthcoming

- Antiferromagnetic effects may be studied shortly

$\therefore$ cross-stimulation with experiment will increase

9) Needed Supporting .efforts

- Computation facilities required are smaller than those needed for equivalent first-principles calculations 
- problems to be tackled by this technique can be however considerably more complex with their attendant large computer requirement

\section{REFERENCES}

1. D. G. Dempsey and L. Kleinman, Phys. Rev. B16, 5356 (1977).

2. D. G. Dempsey, W. R. Grise and L. Kleinman, Phys. Bev. B 18 , 1270 (1978).

3. D. G. Dempsey and I. Kleinman, Phys. Rev. Lett. 39, 1297 (1977).

4. D. C. Des jonqueres and F. Cyrot-Lackmann, J. Phys. F $\underline{7} .61$ (1977) .

5. K. S. Sohn, .. G. Dempsey and L. Kleinman, Phys. Rev. B16, 5367 (1977):

E. CLUSTER MODELS FOR ELECTRONIC STBUCTUEE

R. P. Messmer, I. M. Falicov, J. D. Joannopoulos

1) Problem Area

- To provide a reliable real space description of electronic structure for clean surfaces, chemisorbed species on surfaces, surface defects, and small metal particles

2) Method of Approach

- Electronic structure calculations for finite clusters to describe local environment. These clusters can be either isolated in space or connected to an effective medium

- A variety of theoretical techniques can potentially be applied within this framework: semi-empirical models, e.g. tightbinding approximations to Hartree-Fock theory: first-principles models, e.g.. x-alpha theory, Hartree-Fock theory

3) Long-Term Goals

- To understand for example, the variety of effects observed and obtain reliable information for the following:

- defect regions at surfaces and interfaces

- chemisorption bonds

- the ionic. covalent and metallic character of species at surfaces

- nature of local vibrational surface modes

- binding energies and bond strengths

- nature and importance of atomic and short-range electronic correlation effects

- relation of cluster and extended surface results 
4) Imfact on other Theoretical Research

- Theory of alloys and alloy surfaces

- Photoemission theory, including angle-resolved spectra

- Theory of core shift and shake-up phenomena

- Theory of relaxation phenomena

- Surface vibrational theory

- Excitons

- Adsorbate-adsorbate interactions

- Electron localization-delocalization effects

5) Impact on Experimental Research

- Aid in interpreting all forms of surface spectroscopy

6) Impact on Applied Areas

- The detailed local arrangements of atoms is known to be extremely important in many chemical and metallurgical problems. The cluster methods are very well suited to these questions

- Further the work will be of value in understanding isolated metallic clusters and small particles and stimulating the development and elucidation of concepts needed to understand practical systems

7) Development to Date

- of the first-principles methods, there has been considerable application of the scattered wave $x$-alpha theory to metal clusters and chemisorption systems $[1,2]$

- other first-principles methods which are being developed involve both altcrnative mathematical implementations of $x$-alpha theory [3] as well as ab initio schemes [4,5]

- In the case of semi-empirical methods, there has been extensive application of simplified models to study qualitative trends $[6,7]$

- Some of the few investigations of embedding a cluster in an ef-. fective medium have been carried out through the use of a cluster-Bethe-lattice method combined with an empirical tightbinding model $[8,9]$ 
8) Anticipated Future Development

- There will continue to be activity along establishea lines with applications to many new systems. Furthermore the following developments can be anticipated:

- development of new correlation techniques for local bond effects

- better methods for embedding clusters in effective media

- treatments of vibrational motion and calculation of bond lengths

9) Needed Supporting Efforts

- Availability of substantial computational capability

Additional comments

- Active field of interaction between chemists and physicists

\section{REFERENCES}

1. R. P. Messmer, S. K. Knudson, K. H. Johnson, J. B. Diamond and C. Y. Yang. Phys. Rev. B13, 1396 (1976).

2. R. P. Messmer, in The Nature of the surface chemical Bong. edited by T. N. Rhodin and G. Ertl (North Holland, 1978).

3. O. K. Andersen, W. Klose, and H. Nohl, Phys. Rev. B 17.,1209 (1978):

4. K. Hermann and P. S. Bagus, Phys. Rev. B 17.4082 (1978).

5. S. P. Walsh and W. A. Goddard, Surf. Science 72.645 (1978).

6. D. J. M. Fassaert and A. van der Avoird, Surf. Sci. $\underline{5} \underline{5} 313$ $(1976)$.

7. I. B. Ortenburger, S. Ciraci and I. P. Batra, J. Phys. C 9, 4185 $(1976)$.

8. E. Louis and F. Yndurain, Solid state Comm. 22.147 (1977):

9. R. Laughlin. J. Joannopoulos and D. Chadi. ProC. Int. Conf. on Phys. of sio2 and its Surfaces, IBM. 1978.

F. ELECTRON-SURFACE SCATTERING

S. Y. Tong. F. Jona

1) Problem Area

- Formulation of a one-electron wave functions accurate enough to describe the scattering of positive kinetic energy electrons (continuum states) by surface layers

- calculation of matrix elements and scattering intensities for quantitative comparison with LEED, angle-resolved Auger and XPS, spin polarized LEED, and SEXAFS data 
2) Method of Approach

- The multiple scattering approach in terms of energy-dependent partial wave phase shifts for individual atomic scattering centers is used

- Damped Bloch waves for a finite number of surface layers are are calculated by accumulating solutions in a layer-by-layer scheme

3) Long-Term Goals

- The multiple scattering wave function in the kinetic energy range $10-400 \mathrm{eV}$ finds ready applications in the interpretation of surface spectroscople experiments such as LEED, angleresolved XPS and Auger, spinupolarized LEED, SEXAFS, ELS and angle-resolved UPS, and field-emission

- In some cases (such as angle-resolved UPS) some modification of the final. scattering wave (such as the effect of the core-hole at the erission site) is necessary

- Because of the wide potential applications of the multiple scattering wave function at positive kinetic energies, the problem of finding an accurate and efficient method of generating this wave function is one of the areas central to current theoretical surface research

4) Impact on Other Theoretical Research

- The description of multiple scattering by a continuum state with or without inelastic damping has a wide influence. It has an effect on surface states, band structure, evaluation of excitation matrix elements, etc., calculations

5) Impact on Experimental Research

- Already mentioned in 3

6) Impact on Applied Areas

- Successful interpretation of various surface spectroscopic techniques through the use of an accurate one-clectron multiple scattering wave function could lead to surface parameters such as bond lengths and binding sites important to many applied research areas

7). Development to Date

- The development in this area is one example of recent successes in the theoretical description of experimental data, e.g., LEED intensity voltage (IV) curves

- The multiple scattering approach, in terms of partial wave phase shifts and breaking up a surface region into atomic planes parallel to the surface is used 
- A muffin-tin approximation of the individual scattering potential is employed. This approximation is believed to be valid at the relatively high kinetic energies (>.20 ev above the vacuum level) of the scattering electron

- Layer-by-layer Bloch wave inversion schemes (McRae, Jepsen, Marcus, Jona, et al). angular-momentum space matrix inversion methods (Beeby, Duke, Tong, et al), and the chain method (Pendry and Gard) have been developed (1967-71)

- There was also the development of fast perturbation methods that converges rapidly to the above methods (Pendry, Tong and van Hove) (1973-77). These fast methods allow accurate results to be calculated with savings in computation time by one to two orders of magnitude

- Many surface systems have been analyzed in the area of LEED surface crystallography and in cases where the surface structure was found, very close agreement between the theoretical calculations and experiment was obtained

8) Anticipated Future Development

- one immediate goal is the streamlining of existing computation programs making them usable to other scientific workers. This has becore a necessary step if more theoretical interpretations of experimental data are to be carried out. Similar situations in quantum chemistry (e.g, Mo calculation programs). have found successful ways of distributing computation programs to many workers in the scientific areas

- Another short-term goal is the extension and modification of the multiple scattering LEED wave function to describe, in the oneelectron picture, other areas of surface spectroscopy, such as URS and ELS (electron-loss spertroscopy)

9) Needed Supporting Efforts

- The number of theoretical groups involved in LEED theory has decreased to an alarmingly small number (only 3 groups remain today in this country). Since this area is believed to be important to many practical applications as well as other areas of theoretical and experimental research, it is vital that the computation programs so far developed be made widely available. There is a real danger that some of the knowledge gathered and techniques developed so. far may be lost if. in a couple of years, the theoretical activities in this area are allowed to be further decreased

- It is our opinion that this area urgently needs the stimulus and active participation of new talents 
G. MANY-ELECTRON EFFECTS

P. Feibelman, L. Kleinman

1) Problem Area

- Evaluation of many-electron effects in static and dynamic surface properties

2) Method of Approach

- Isolation of many-electron effects from complications introduced by the crystal lattice -- the jellium approximation

3) Long-Term Goals

- Intrinsic properties of surfaces:

- development of a reliable. practical means for incorporating exchange and correlation effects into surface electronic structure calculations

- understanding the nature of electron transfer in chemisorptive bonding

- determining the role of electron collective motion in surface eneryies

- determining properties of electronic elementary excitations of surfaces and their coupling to charged particles

- Analysis of experiments that probe surfaces :

- development of a reliable, practical description of nonlocality of surface dielectric response

- understanding effects of nonclassical electromagnetic fielas in optical excitation of surface electrons in photoemission, ellipsometry. Raman scattering, etc.

- determining deviations from the image force as a function of incident particle momentum near a surface-evaluation of the effects of these deviations in electron-vibrational excitation. LEED, ion diffraction, tunneling spectroscopy. field emission. etc.

4) Impact on Other Theoretical Research

- Ability to use local XC potentials in realistic surface electronic structure calculations

5) Impact on Experimental Research

- Interpretation of yield vo frequency in photoemission of surface electrons into vacuum or electrolyte (elimination or at least understanding of which features are due to dielectric response)

- Interpretation of surface reflection data using p-polarized light.

- What causes the enhanced Raman effect seen in , for example. Pyridene on Ag 
- Interpretation of beam Intensities in electron excitation of surface vibrations

- Interpretation of tunneling spectroscopy -- role of deviations from the image force

- Bole of electron-hole excitations in damping diffractive ionsolid scattering

7) Development to Date

- Intrinsic surface properties:

- Lang and Kohn, demonstration that work functions can be calculated reasonably accurately using local XC potential

- discovery that screening charge likes to occupy atomic-like orbitals on adatoms having unfilled shells but not on those with filled shells (Lang and williams)

- studies showing surface plasmon properties are very sensitive to surface structure (Feibelman)

- Analysis of surface experiments:

- demonstration via jellium calculations that electromagnetic fields near surfaces are highly nonclassical, strongly frequency dependent, rapidly varying in space (Feibelman, Kliewer)

- semiclassical evaluations of deviations from image force (Mills, Rudnick. Feibelman, Duke and Bagchi. etc.l

8) Anticipated Future Development

- Much of the work in this field is done and the important steps to be taken involve inclusion of the crystal lattice. Probably the most important area in which jellium work shoula continue is in the analysis of time-evolution effects in electron emission experiments.

H. PHOTOEMISSION THEORY

P. Feibelman, W. Plummer, S. Y. Tong

1) Problem Area

- Develop theory. for the analysis of experimental photoemission results

2) Method of Approach

- Attempt to push one-electron calculations to the limit to assess their adequacy for the analysis of photoemission data

- spatial variation of electromagnetic field in the surface region must be included 
3) Long-Term Goals

- Ability to use photoemission data to analyze surface bonding. electronic structure and atomic geometry

- Ability to recognize when correlaticn effects are essential to the analysis of photoemission data

4) Impact on Other Theoretical Research

- The questions posed in analyzing photoemission data arise in the analysis of many other surface experiments

- The choice of final state wave function for example is important in LEED, SEXAFS and angle-resolved XPS

- The question of how to treat the near surface electromagnetic field is significant in ellipsometry. surface reflection spectroscopy and surface Raman spectroscopy

5) Impact on Experimental Research

- Directly connected

7) Development to Date

- LEED wave functions have been used for final electron states

- The inclusion of the elecliostatic attraction of the hnle left behind by the photoelectron has been studied

- First attempts to use initial and final wave functions appropriate to the same potential have been made, both for slab and cluster geometry.

- To date correspondence between photcemission calculations ann dat.a is poor: both the observed widths and dispersion of surface peaks are hard to rationalize

- Jellium calculations of the near surface fiela have been carried out. They show that this field is highly nonclassical, spatially rapidly varying and strongly frequency dependent. Contact with experiment is lacking.

8) Anticipated Future Development

- Development of a means for taking the effects of hole localization into account consistently in the initial and final electron wave functions

- Studies of the importance of removing the muffin-tin approximation and including a self-consistent surface potential barrier and the image force in evaluating the final state wave function 
- Development of a practical approximation for the spatial and frequency behavior of the near surface electromagnetic field

9) Needed Supporting Efforts

- More experimental data

I. CORE-LEVEL EXCITATIONS

W. Spicer, R. P. Messmer, P. J. Feibelman

1) Problem Area

- The origin of energy shifts of core levels in photoemission spectroscopy. especially as they arise.from surface rearrangement or chemisorption of species at the surface. is poorly understood

- Effects such as relaxation, core-hole screening, shake-up and excitonic interactions produce additional structures and in some cases can shift the core peak in photoemission spectra

- It may be possible to obtain quantitative information about chemisorpticn bonding from core-level spectra

2) Method of Approach

- Theoretical methods which treat core electrons of the atoms and are based on a localized viewpoint. One-electron methods should be applied using "first-principles" approaches to determine the limitations of one-electron theory

- Extensions of present one-electron first-principles theories should be made to include correlaticn effects which are probably necessary to understand the problem completely

- Time-dependent simple model Hamiltonians should be used to investigate dynamics of core holes

3) Long-Term Goals

- Must understand core-level lineshapes in order to determine meaningful experimental lineshifts

- To learn if lineshifts can be used to determine meaningful chemical and physical information

- With respect to above point, it is necessary to understand the degree to which the lineshift can be decomposed into chemical and dynarical relaxation shifts 
- Ability to identify multiplet effects in core shifts

4) Impact on Other Theoretical Research

- Related to correlation effects in photoemission spectra from narrow bands

- Related to time evolution of nonstationary many-electron states

5) Impact on Experimental Research

- Interpretation of photoemission data

- Interpretation of Auger processes

- Ability to distinguish shake-up frum one-electron frocesses in "fingerprinting" of surface species in photoemission or Auger

6) Impact on Applied Areas

- Semiquantitative use of core level spectroscopies in applied research is already.widespread, so improved interpretation could be immediately useful

7) Development to Date

- Qualitative understanding of screening effects in XPS has been established by using jellium calculations. This picture permits a division into dynamic and chemical shift terms for the tutal shifts

- Excited-atom models for relaxation combined with self-consistent band structure for chemical shitt have predicted erends in shifts reasonably well for bulk metals

- A variety of effects producing satellites has been identified and systematics are being identified

- Infrared singularity effects in XPS lineshapes have been treated successfully by a simple parameterized model to give qualitative understanding of lineshapes

8) Anticipated Future Development

- More detailed one-electron shift calculations

- Studies of time-dependent schroedinger equation using simple model Hamiltonians and incorporating core-hole lifetime

- Similar studies using more sophisticated (first krinciples) models 
J. EXTENDED VS LCCALIZED ELECTRON STATES

W. Plummer. J. Joannopoulos, M. Schluter

1) Problem Area

- The problem is to develop a new theoretical formalism capable of describing extended one-electron states and localized correlated many-electron states in a unified framework

- Many chemisorption systems and excitation experiments are now known to exhibit features which require this type of combined formalism

2) Method of Approach

- This is really a new theoretical direction which requires a different new approach, which cannot be specified by us

3) Long-Term Goals

- The objective is to create a theory which allows one to unaerstand the interplay of extended and localized states. This should be applied to understand the ground state total energy of surfaces and adsorbates

- There are several experimental observations which demand the creation of such a theoretical formalism. For example, linewidths, energy dispersion and shape-up energies and intensities in the excitation of a valence electron of an adsorbed atom or molecule cannot be treated in the extended or localized one-electron picture

- Photoemission excitation of the valence bands of metals like $\mathrm{Ni}$ show multi-electron excitations. An overall goal should be a complete theory of the time evolution in an excitation process

4) Impact on Other Theoretical Research

- This problem requires a completely new formalism which if developed could have far-reaching impact on theoretical research

5) Impact on Experimental Research

- A theoretical understanding of this localized-delocalized problem will have direct impact on nearly all excitation experiments

- The interpretation of core and valence level shifts as shake-up intensities of adsorbates, and the bulk excitation of metals like Ni depend upon an understanding of this problem 
6) Impact on Applied Areas

- There are several applied areas like mos junctions where the defect problem could be treated by such a theory

7) Development to Date

- configuration interaction calculaticns of small molecules are able to describe localized correlated electronic states but are unable to describe extended states beyond the size of the cluster

- Hubbard-type calculations have been restricted to very simple Hamiltonians

- Ad hoc separation of localized and extended states has been treated in tight-binding calculations

K. POSITRON-SURFACE. INTERACTIONS

M. Schluter

1) Problem Area

- positron annihilation in surface studies, and associated theoretical aspects

2) Method of Approach

- calculation of positron energies in a background of metallic surface electrons. Positrons are treated adiabatically and not as quantum particles

3) Lonq-Term Goals

- Nonadiabatic, self-consistent calculation of $\mathrm{N}$-electron +1 positron surface system

- Evaluation of dynamical correlation effects

$\therefore$ Development of positrons as an independent surface probe complementary to electrons

4) Impact on other Theoretical Research

- A dynamical theory of electron-positron correlation is strongly tied to aspects that arise in any many-body system 
5) Impact on Experimental Research

- The understanding of the behavior of positrons (and positronium) on surfaces could lead to the development of "slow" positron sources

7) Development to Date

- The theory to date suffers from the nonself-consistent treatment of electron and positron and from the adiabatic description of the positron

- Within these limits, model potentials and work functions for positrons on metals with varying electron density have been constructed. Comparison to experiment is fair

8) Anticipated Future Development

- Formulation of adequate many-particle theory (possibly in a local description) and self-consistent integration of the equations to yield reliable results for bound state energies and work functions

9) Needed Supporting Efforts

- Workable many-body theory for positron in electron environment 
VII. PBOBLEM AREAS - GENERAL

A. SOLID-ELECTBOLYTE INTERFACES

P. Schmidt, w. Spicer

1) Problem Area

- The solid-electrolyte interface figures importantly in electrochemical charge transfer. photoelectrochemistry. heterogeneous catalysis

- The structure of this region and the nature of the chemical and physical interactions are only incompletely understood

2) Method of Approach

- Theoretical investigations require the application of statistical and molecular quantum mechanics

- In adaition, methods of the solid state physics of metals and semiconductors are needed frequently to account for the effect of the physical boundary in this clearly complicated. composite region

3) Long-Term Goals

- Knowledge of the structure of the interfacial state is important and should be sought

- More needs to be known about the structure of reactive molecular species in the interface. It is important to understand how molecular species undergo changes in geometry as they migrate from the bulk of the solution to the interface

- A study of the molecular structures implies an examination of the stationary state energetics of these species. The stationary energy. states are essential to any examination of chemical reactions in the interfacial region

- Spectroscopic methods can provide valuable information about interfacial molecular structure and stationary state energies. It is important therefore to determine how the structure of the interfaces. and in particular the metal-solution boundary. influences the absorption and emission of light

- The electromagnetic field can directly influence chemical reactivity in the interface. photoelectrochemistry and photocatalysis are relatively new experimental areas. To date these areas have not received enough attention from theorists 
4) Impact on other Theoretical Research

- It is essential to know the structure, and by implication the quantum mechanical and thermodynamic stationary states, of species in the interface before any attempt can be made to examine chemical reaction kinetics in this region

- The general theoretical descriptions of electrochemical reactions, and essentially catalysis, require a good understanding of the molecular stationary states of reactants in the interface

5) Impact on Experimental Research

- Structure assumptions often imply definite chemical interactions and reactivities. These can be tested directly by experiment

- Photochemical interactions can involve predictions of the appearance of specific chemical species

6) Impact on Applied Areas

- There is already considerable research support for experimental electrochemistry. The search for new energy sources and energysaving electrochemically-based technologies is active

- Theoretical investigations which could lead to more efficient, cost saving practical investigation could be of immense value. At present, much of the practical, technological effort to find these energy savers are "hunch-based"

7) Development to Date

- There is a vast amount of statistical mechanical analysis of the electrical interfacial double layer. Much more can be done with respect to the application of formal statistical mechanical methods

- Practically nothing has been done yet on the theoretical level to investigate the molecular structure and stationary states in the interface.

- There has been some work done on electromagnetic field problems, but more needs to be done

8) Anticipated Future Development

- There has been too little theory at the atomic and electronic structure level to anticipate short-term developments 
B. ALLOY SURFACES

L. M. Falicov, T. E. Fischer

1) Problem Area

- Atomic composition

- Segregation. short- and long-range order effects

- Thermodynamics

- Electronic structure and states

- Chemical properties

2) Method of spproach

- Phenomenological approach: Coordination of data from alloy and thermodynamic tables

- Semiphenomenological approach: Input of atomic interactions and calculation of thermodynamic variables

- First principles: Electronic calculations with a real-space, local approach

3) Long-Term Goals

- Determine surface composition and stability in vacuo and in diverse environments

- study electronic structure of alloy surfaces

- Study magnetic properties of alloy surfaces

- Determine charge distributions, charge transfers, "ionicity" and "Covalency" in alloy surfaces

- Understand reactivity of alloy surfaces

- Tailor surfaces in regard to corrosion and chemical properties, and catalysis (in particular selectivity)

4) Impact on other Theoretical Research

- Possible impact in understanding magnetism and phase transitions at elemental surfaces

- contribution to the understanding of localization effect at surfaces in general 
5) Impact on Experimental Research

- self-evident

6) Impact on Applied Areas

- It has the potential for producing new materials in heterogeneous catalysis

- Grain boundary segregation is known to have a large effect on strength of materials and its change under various environments (e.g.. temper embrittlement, hot shortness, cavitation, creep. etc.). These effects will gain in importance as metal recycling increases

7). Development to Date

- Phenomenological approach under control but just beginining to be exploited $[1,2,3]$

- Semiphenomenological approach very active at this time and making good progress [4,5]

- First-principles approach in its infancy

8) Anticipated Future Development

- Phenomenological approach will continue in its high productivity stage, with high technological impact

- Semiphenomenological approach development will accelerate appreciably

- First-principles theory may begin tc develop: Hard work will be required

9) Needed Supporting Efforts

- strong interaction with experiment is paramount. crossmonitoring of theoretical and experimental efforts are a must

- Other theoretical efforts (e.g., localization problem) may influence direction of research

\section{REFERENCES}

1. D. McLean, Graiin Boundaries in Metalals (Oxford University Press, London, 1956).

2. J. J. Burton and E. S. Machlin, Phys. Rev. Lett. 3 ? ,1433 $(1.976)$.

3. C. J. McMahon, Jr. and L. Marchut, J. Vac. Sci Technol. $1 \underline{5}, 450$ (1978). 
4. M. Guttmann, Surf. Science .su 53,213 (1975).

5. J. L. Moran-Lopez and L. M. Falicov. Rhys. Rev. B 18, 2542,2549, and 2555 (1978).

C. ELECTRON STIMULATED DESORPTION

P. Feibelman

1) Problem Area

- Chemisorbed atoms desorbed by electron-beam produced excitations display threshold effects as a function of beam energy

- Anisotropic velocity distributions are found for the desorbed atoms or ions

2) Method of Approach

- Theory is relatively primitive: attempt to correlate prominent experimental results such as strong angular anisotropy ard threshold observations to surface bonding site, bonding angles and valency.

3) Long-Term Goals

- Determination of what aspects of the mechanism of ESD are responsible for the angular anisotropy and energy distributions which are observed, via development of a ccattering theory. of the process

- Elucidation of the role of ionicity vs covalency in the Auger decay mechanism for ESD

4) Tmpact on other Theoretical Research

- Strong observed valence sensitivity of ESD suggests that we can deepen our understanding of the theoretical meaning of valency by developing ESD theory

- Theory of reneutralization of ions will bear on ion neutralization spectroscopy

5) Impact on Experimental Research

- Main thrust of theory is interpretation of experiments

- It is not clear that ESDIAD (Ion angular Distribution) patterns actually reflect initial state bond angles. Demonstration that this is true would make ESDIAD very useful 
6) Impact on Applied Areas

- ESD is the process by which compcund surfaces decompose in ionizing environments. Further elaboration of the systematics of ESD vs valency may help in the development of materials that resist such decomposition

7) Development to Date

- It has been shown that ESD threshold phenomena can be explained by assuming that the initial steps in desorption are the creation of a core-hole and its subsequent Auger decay. This mechanism implies that ESD thresholds indicate the bonding site of the desorbing species and the valency in the unperturbed state. The simplicity of obtaining useful surface information from ESD data suggests that the experiment will become more popular

- Models of ESDIAD show that if interference between vibration and the motion which leads to desorpticn can be ignored, as well as any anisotropy in the reneutralization process, then re-analysis of ion-angular distributions can yield bonding angles and vibration frequencies

8) Anticipated Future Development

- studies of the time evolution of a desorption event will be carried out to establish the degree to which initial state bonding is reflected in ESD angular and energy distributions

D. EMPIRICAL MODELING OF CHEMICAL TRENDS IN SURFACE PHENOMENA

T. E. Fischer, R. Messmer, W. E. Spicer

1) Problem Area

- The elaboration of a number of simple empirical rules codifying trends in the variation of surface properties through the periodic takle would be useful, particularly in solving practical problems

- What is wanted is a number of models that are approximations to, or the expression of results of, complex theories of surfaces

- Enough theoretical insight and experimental information are available so that the construction of such rules should now be possible. 


\section{2) Method of Approach}

- Based on the results of theoretical calculations and of measurements, one estimates how a given quantity varies from one substrate to another for a given adsorbate or from one adsorbate to another for a given substrate. One seeks to express this variation quantitatively in terms of one or more known quantifiable properties of the substrate or adsorbate. Variations with the periodic table will be useful as a starting point

3) Long-Term Goais

- A set of rules that codify knowledge acquired by theory and experiment in a set of simple formulae with reasonable quantitative accuracy

4) Impact on Other Theoretical Research

- Empirical trend́s may lead theorists to recognize and explore previously ignored relationships or suggest fruitful problem areas

5) Impact on Experimental Research

- Trend rules will assist in the interpretation of experimental data, especially if these rules are based on more rigorous theory

- They w1ll suggest new systems (chemical compositions) to be explored in order to achieve a desired effect or check theoretical predictions

- They will challenge experfmentalists to check given trend models

6) Impact on applied Areas

- Impact will be greatest here. These trend rules will save vast amounts of time in the search for novel or better adapted catalysts and in the invention of devices or processes in various areas.

\section{7) Development to Date}

- Such rules have existed for a long time and have been useful for bulk properties (e.g.. Pauling rules of chemical bond, ErigelBrewer rules for intermetallic compounds, philips dielectric theory of semiconductors)

- scme empirical rules exist in catalysis. (the "volcano curve" relating catalytic activity to binding energy of adsorbates and the correlation of present d character of substrate cohesion). These rules are too ill defined and simple and are of limited use 
- A promising start is the use of the new spin-orbital electronegativity concept [1] as an aid to prediction of chemical reactivity

8) Anticipated Future Development

- As the sophisticated theories relating electronic structure. chemical reactivity and local bond strengths are used to produce quantitative information on important sample systems, one should encourage and can expect the appearance of trend rules that are suited for use in solution of Eractical problems by people not familiar with complex theories

9) Needed Supporting Efforts

- General development of surface science

- Accumulation of experimental data

- Quantitative, reliable theories

\section{REFERENCES}

1. K. H. Johnson, J. Vac. Sci. Technol. 15 ;478 (1978). 
VIII: INNOVATION IN SURFACE SCIENCE

The panel addressed the question of innovation in surface science, initially posed in the following form: Given the fact that innovations cannot be predicted, could we at least guess something about the general form that a real innovation might take in our field? The consensus was that we could not do so in general; but that several questions posed in our technical discussions demanded completely innovative solutions. (These were the local-nonlocal hole state problem area, and the equal time scale: or breakdown of adiakaticity problems discussed in several problem areas.)

The panel next considered whether the kind of discontinuity i.n knowledye represented by such examples from bulk solid-state physics as the BCS theory or wilson's critical puint calculations could be expected in surface science. It was concluded that both of these problems were "ripe" for solution in the sense that accurate empirical models and relationships correlating large amounts of data existed. No comparable systemization of data yet exists in a problell area in surface science.

It was pointed out that the reduction of surface structural determination to a routine matter would set the stage for great advances in our understanding. Firally. the manner in which theory can stimulate innovation in applied surface areas by directing empirical searches was stressed. 
IX. INSTITUT IONA I ISSUES

A number of questions concerning the organization and support of research were raised in the course of the meeting. and were reviewed in the final session. That discussion is summarized here.

A. Basic vs Applied Reșearch

Pioneers in surface science like Davisson, Germer, and langmuir made great scientific advances while pursuing practical goals. The separation between basic and applied research is relatively new. The problem of the separation starts at the educational level, where a physics graduate student headed for solid-state research may often end up taking more courses in high-energy physics than in chemistry. despite the fact that the latter is much more important to him. The importance of breaking down this separation should be realized.

B. Promotion of Innovation

There is a widespread tendency for researchers to work mainly on problems that are small extensions of probiems already solved. This conservatism is fostered by two-year contract renewal periods. The situation could be improved by five-year grant renewal periods, or special grants for an estabiished researcher to take a major risk or switch fields to try a new idea without cutting himself off.

C. Supply of Young scientists

The enrollment of physics and chemistry graduate students is falling off markedly. since careers in research no longer seem attractive. This is due at least in part to the difficulty of a young person getting started and the long time scale to a permanent (academic) position. A DOE sloan-type fellowship funding promising young people with no strings could aid here. An additional factor specifically discouraging graduate study in theory is the fact that most industrial research laboratories do not hire theorists. The same institutions have been hiring a significant number of PhD's with experimental surface science training. Increased general awareness of the contributions of theory to surface science may have an effect here. but the panel has no specific froposals.

D. New surface science centers

The 1977 workshop report proposed the creation of new research centers for surface science. This panel was not in favor of this proposal, citing the following reasons: 
- It would institutionalize proklems that now exist

- The diverse milieus in which most of us now exist are much more stimulating than a focused institute, which could de a deadening environment

- The National Center for Computational chemistry was cited as an example of a concept that was outmoded before it was functioning

- It is feared that control of such a center would be too political

- Fioxibility and dynamic response to new ideas are very important and are better achieved with scattered, indepcndent researchers

E. Critical Mass and Communication

- It was estimated that at most 50 us scientists are involved in theoretical surface science at present. Since the panel began its problem area compilation with a list of over 50 topics. this spreads efforts rather thinly at best

- Problem areas involving major computational development like LEED and electronic structure especially need mutual stimulation and cross-checking. The number of groups developing LEED theory has fallen dangerously low, and electronic structure is only llaryirally above a critical level.

- Interdisciplinary communicaticn is seen as improving. with surface science stimulating lie development. Tt is difficult to foster such an increase from the outside; such communication grows as it is perceived to be advantageous to individual scientists

- Joint research proposals involving different aisciplines have difficulties in being funded because the funding agencies are not set uf to deal with them. provision should be made to du so, and sucli piopnals should he specifically encouraged.

F. Surface Theory Outside the USA

- There is considerable activity in basic surface science, both experimental and theoretical, in Western Europe. The USSR has no significant program in suríace science as such, but leads the world in theoretical electrochemistry. Japan has no significant suriace theory effort.

- Three areas of surface theory representing significant efforts in Europe are electronic structure, many-body effects and LEED. Electronic structure studies have been extensively pur- 
sued in England and France. English work has concentrated on surface states of abruptly terminated crystals, on innovative methods for solving tight-binding models, and on simple models for single-atom chemisorption. Work in France has concentrated on simple tight-binding models for transition metal surfaces. A group is active in spain which primarily derives from the English school. As a whole, the European workers in this area have stressed elegant approaches to simpler models compared to US workers, whose emphasis has been on more detailed realistic models. There have been some realistic cluster calculations in Germany, and a start has been made toward competing with US workers on extended surface calculations in Denmark.

- In LEED, English workers have contributed significantly in the development of current theory, but are not especially active in pursuing structure determinations at present. Some French workers are entering this area. The general quality of the European LEED theory is about on a par with that in the US. Related work cn angle-resolved photoemission is being pursued in Germany.

- In many-body effects, especially as related to electron excitations important in various surface spectroscopies, groups. in Sweden and Denmark are leading contributors. Nc comparable effort exists in the US. 